\title{
A Protein Interaction Node at the Neurotransmitter Release Site: Domains of Aczonin/Piccolo, Bassoon, CAST, and Rim Converge on the N-Terminal Domain of Munc13-1
}

\author{
Xiaolu Wang, ${ }^{1}$ Bin Hu, ${ }^{1}$ Agata Zieba, ${ }^{2}$ Nicole G. Neumann, ${ }^{2}$ Monika Kasper-Sonnenberg, ${ }^{1}$ Annegret Honsbein, ${ }^{2}$ \\ Greta Hultqvist, ${ }^{2}$ Tim Conze, ${ }^{1,2}$ Wolfgang Witt, ${ }^{1}$ Christoph Limbach, ${ }^{2}$ Matthis Geitmann, ${ }^{3}$ Helena Danielson, ${ }^{3}$ \\ Richard Kolarow, ${ }^{4}$ Gesa Niemann, ${ }^{4}$ Volkmar Lessmann, ${ }^{4,5}$ and Manfred W. Kilimann ${ }^{1,2}$ \\ ${ }^{1}$ Institut für Physiologische Chemie, Ruhr-Universität Bochum, D-44780 Bochum, Germany, Departments of ${ }^{2}$ Neuroscience and ${ }^{3}$ Biochemistry and \\ Organic Chemistry, Uppsala University, S-75124 Uppsala, Sweden, ${ }^{4}$ Institute for Physiology, University of Mainz, D-55128 Mainz, Germany, and 5 Institute \\ of Physiology, Otto-von-Guericke University, D-39120 Magdeburg, Germany
}

\begin{abstract}
Multidomain scaffolding proteins organize the molecular machinery of neurotransmitter vesicle dynamics during synaptogenesis and synaptic activity. We find that domains of five active zone proteins converge on an interaction node that centers on the $\mathrm{N}$-terminal region of Munc13-1 and includes the zinc-finger domain of Rim1, the C-terminal region of Bassoon, a segment of CAST1/ELKS2, and the third coiled-coil domain (CC3) of either Aczonin/Piccolo or Bassoon. This multidomain complex may constitute a center for the physical and functional integration of the protein machinery at the active zone. An additional connection between Aczonin and Bassoon is mediated by the second coiled-coil domain of Aczonin. Recombinant Aczonin-CC3, expressed in cultured neurons as a green fluorescent protein fusion protein, is targeted to synapses and suppresses vesicle turnover, suggesting involvements in synaptic assembly as well as activity. Our findings show that Aczonin, Bassoon, CAST1, Munc13, and Rim are closely and multiply interconnected, they indicate that AczoninCC3 can actively participate in neurotransmitter vesicle dynamics, and they highlight the N-terminal region of Munc13-1 as a hub of protein interactions by adding three new binding partners to its mechanistic potential in the control of synaptic vesicle priming.
\end{abstract}

\section{Introduction}

Neurotransmitter release is confined to a specialized area of the presynaptic plasma membrane known as the "active zone." Calcium-dependent exocytosis at the synapse is an elaborate, multistep process that is fast and precise yet highly restrained and subject to modulation (presynaptic plasticity) (Wojcik and Brose, 2007). Moreover, it is functionally coupled to re-endocytosis, which maintains the homeostasis of presynaptic membranes by the retrieval of proteins and lipids into the vesicle compartment. A cytoskeletal-like, solubilization-resistant lattice of proteins associated with the inner face of the presynaptic plasma membrane, the cytomatrix at the active zone (CAZ), is apparent in electron microscopy and seems to integrate and organize many proteins of the presynaptic machinery. However, our understanding of the precise molecular architecture of active zones and of the mechanistic steps through which their molecular constituents interact is still rudimentary (for review, see Gundelfinger et al., 2003; Ziv and Garner, 2004; Schoch and Gundelfinger, 2006).

\footnotetext{
Received March 8, 2009; revised Aug. 19, 2009; accepted Aug. 21, 2009.

This work was supported by grants from Deutsche Forschungsgemeinschaft, Fonds der Chemischen Industrie, the intramural research funding program (FoRUM) of the University of Bochum Medical School, and startup funds from the Faculty of Science and Technology of Uppsala University (M.W.K.).

Correspondence should be addressed to Manfred W. Kilimann, Department of Neuroscience, Box 593, Uppsala University, S-75124 Uppsala, Sweden. E-mail: manfred.kilimann@neuro.uu.se.

X. Wang's present address: Evotec AG, NeuroScience, D-22525 Hamburg, Germany.

DOI:10.1523/JNEUROSCI.1255-09.2009

Copyright $\odot 2009$ Society for Neuroscience $\quad$ 0270-6474/09/2912584-13\$15.00/0
}

Five large multidomain proteins, or small protein families, have been identified that are believed to be important for the organization and functioning of the molecular machinery of mammalian active zones, both during the assembly of synapses and in acute vesicle dynamics: Aczonin/Piccolo (550 kDa), Bassoon (420 kDa), CAST/ERC/ELKS ( 100 kDa), Munc13 $(\sim 190 \mathrm{kDa})$, and Rim $(\sim 170 \mathrm{kDa})$. Several physical interactions of these five CAZ proteins with other proteins have been identified. These include interactions between each other (Munc13 with Rim; Rim with CAST; CAST with Bassoon and Aczonin), interactions with other proteins of presumed scaffolding functions (Munc13 with $\beta$-spectrin III $\Sigma 1$; Aczonin with GIT; Bassoon with CtBP1/Ribeye; CAST with liprin- $\alpha$ and syntenin-1; Rim with Rim-BPs, 14-3-3, and liprin- $\alpha$ ), and interactions with proteins involved in vesicle traffic or fusion (Rim with Rab3, Rab6, synaptotagmin, SNAP-25, and the $\alpha 1 \mathrm{~B}$ calcium channel subunit; Munc13 with syntaxin and DOC2; Aczonin and Bassoon with PRA1), in cytoskeletal remodeling (Aczonin with profilin and Abp1), or in synaptic plasticity (Rim with cAMP-GEFII; Munc13 with calmodulin) (for references, see Schoch and Gundelfinger, 2006). Numerous additional interactions can be expected to be found, given the large sizes and multidomain architectures of the proteins involved. The emerging picture is that of a highly interwoven protein network, and the giant proteins Aczonin and Bassoon are presumed to be of particular importance as scaffolds for the structural and functional integration of the protein machinery of the active zone. 
In the present study, we have identified several new molecular interactions among the CAZ proteins Aczonin, Bassoon, CAST1, and Munc13-1. Strikingly, in addition to the known binding of the Rim Zn-finger domain, several interactions converge on the $\mathrm{N}$-terminal region of Munc13-1, highlighting it as a potential hub for multilateral crosstalk between these proteins and suggesting a high degree of supramolecular organization at the neurotransmitter release site.

\section{Materials and Methods}

cDNA sequences and vectors. The complete coding cDNA sequences of mouse CAST1, Munc13-1, and Rim1 were amplified in overlapping segments from mouse brain RNA by reverse transcription (RT)-PCR, initially with rat primers. Sequences, including multiple splice variants of CAST1 and Rim1, were deposited in GenBank under accession numbers AY356530-8 and AY753536. Glutathione S-transferase (GST) fusion proteins were expressed from the vector pGEX-4T-2 (SmaI site; GE Healthcare). Fusion proteins with $\mathrm{N}$-terminal His tags were expressed from pQE32 (SmaI site; Qiagen). Constructs with an N-terminal thioredoxin fusion and a C-terminal His tag were in pBAD/Thio-TOPO (Invitrogen). Construct Mul:pet and the series Mul + 2-1 to Mu1 + 2-6 were cloned into a derivative of pET3 (Novagen) engineered in our laboratory such that the recombinant protein begins with the natural start codon of Munc13-1 and carries a C-terminal His tag. Enhanced GFP (EGFP) fusion constructs were cloned into the SmaI site of pEGFP-C1 (Clontech).

Antibodies. cDNAs encoding Aczonin, Bassoon, CAST1, Munc13-1, and Rim 1 partial sequences were amplified from mouse brain RNA by RT-PCR and inserted into the SmaI sites of the His-tagged vector pQE32 (Qiagen) or the GST vector pGEX-4T-2 (GE Healthcare). Recombinant proteins were expressed in bacteria and used for the immunization of rabbits and guinea pigs. The following constructs were used as antigens: AczN (amino acids 63-218, LPK... IPK), Aczp18p19 (amino acids 1808-2150, QKE ... GVS), Cast4a (amino acids 711-957, EAS ... IWA), Rim43 (amino acids 9-387 $83-105$, GPR ... RYP), and Mu1 [amino acids 2-320, SLL ... QDE, as a thioredoxin fusion protein in $\mathrm{pBAD} /$ Thio-TOPO (Invitrogen)]. Sera were affinity purified on the same recombinant proteins used for immunization. Anti-Aczonin immunoprecipitations (IPs) and Western blot developments were performed with anti-Aczp18p19 unless indicated otherwise. A mouse monoclonal antibody against rat Bassoon (amino acids 738-1035) was obtained from Nventa Biopharmaceuticals. A Rim1 monoclonal antibody (clone 26; immunogen, amino acids 602-723 of rat Rim1) was from BD Biosciences ("Rim1-BD"), and Munc13 monoclonal antibodies were purchased from BD Biosciences [clone 32; immunogen, amino acids 621-834 of rat Munc13-1 ("Munc13-BD"); described by the vendor as panMunc13 reactive] and Synaptic Systems [immunogen, amino acids 1399-1736 of rat Munc13-1 ("Munc13-SS"); described by the vendor as Munc13-1 specific]. A rabbit antiserum against neurobeachin (Nbea) has been described previously (Wang et al., 2000). Most other antibodies were purchased from BD Biosciences.

Immunoprecipitation. Mouse brains were homogenized with a glassTeflon homogenizer in $5 \mathrm{v} / \mathrm{w}$ of IP lysis buffer (20 mM Tris, $\mathrm{pH} 7.4,150$ mм NaCl, 0.5 mм EDTA, 1 mм Na $\mathrm{VO}_{4}, 10 \mathrm{~mm} \mathrm{NaF}, 2$ mм PMSF, 2 $\mu \mathrm{g} / \mathrm{ml}$ pepstatin A, $2 \mu \mathrm{g} / \mathrm{ml}$ leupeptin, $2 \mu \mathrm{g} / \mathrm{ml}$ aprotinin, $2 \mathrm{~mm}$ benzamidine, $0.5 \%$ Triton X-100, and $0.5 \%$ BSA) and centrifuged at $120,000 \times$ $g$ and $4^{\circ} \mathrm{C}$ for $30 \mathrm{~min}$. Supernatants were precleared with $1 / 60$ vol of Pansorbin (Calbiochem) at $4^{\circ} \mathrm{C}$ for $1 \mathrm{~h}$. Aliquots $(400 \mu \mathrm{l})$ of precleared lysate were incubated at $4^{\circ} \mathrm{C}$ for $1 \mathrm{~h}$ with 5-10 $\mu \mathrm{l}$ of crude sera, 5-10 $\mu \mathrm{l}$ of monoclonal antibodies, or 50-100 $\mu$ l of affinity-purified sera. A total of $60 \mu \mathrm{l}$ of $10 \%$ Pansorbin (preblocked with $3 \%$ BSA in IP lysis buffer at $4^{\circ} \mathrm{C}$ for $1 \mathrm{~h}$ ) were added, and samples were incubated with rotation for an additional hour. Pansorbin was sedimented at $10,000 \times g$ for $1 \mathrm{~min}$, and pellets were washed five times with $1 \mathrm{ml}$ of IP lysis buffer and analyzed by SDS-PAGE and Western blotting.

Brain lysate pull-down assay. Rat brains were homogenized with a glass-Teflon homogenizer in pull-down tissue lysis buffer (50 mм Tris, pH 7.4, 150 mм NaCl, 4 mм $\mathrm{MgCl}_{2}$, 0.5 mм EDTA, 1 mм DTT, 0.5\% Triton X-100, $0.5 \mathrm{~mm}$ PMSF, and $2 \mu \mathrm{g} / \mathrm{ml}$ each of pepstatin A, leupeptin, and aprotinin) and centrifuged at $120,000 \times g$ and $4^{\circ} \mathrm{C}$ for $30 \mathrm{~min}$. Supernatants were precleared with $1 / 10 \mathrm{vol}$ of glutathione agarose beads (prewashed with pull-down lysis buffer) for $4 \mathrm{~h}$ at $4^{\circ} \mathrm{C}$ and adjusted to a protein concentration of $10 \mathrm{mg} / \mathrm{ml}$ with pull-down lysis buffer. Glutathione agarose beads were loaded with GST fusion proteins or unmodified GST $(\sim 20 \mu \mathrm{g}$ of protein per $20 \mu \mathrm{l}$ of beads), preblocked with $3 \%$ BSA in lysis buffer for $2 \mathrm{~h}$ at $4^{\circ} \mathrm{C}$, and washed with $1 \mathrm{ml}$ of lysis buffer. Aliquots $(20 \mu \mathrm{l})$ of loaded beads were incubated with $300 \mu \mathrm{l}$ aliquots of precleared brain lysate at $4^{\circ} \mathrm{C}$ overnight under end-over-end agitation. Beads were spun down, washed rapidly with $3 \times 1 \mathrm{ml}$ of ice-cold lysis buffer, boiled in SDS-PAGE sample buffer, and analyzed by Western blotting using the chemiluminescence detection system. His-tagged fusion proteins were immobilized for pull-down assays by covalent coupling. Affi-Gel 15 (40 $\mu \mathrm{l}$; Bio-Rad) were incubated with $200 \mu \mathrm{l}$ of fusion protein $(0.4-1 \mathrm{mg} / \mathrm{ml})$ in coupling buffer ( $100 \mathrm{~mm} \mathrm{NaCl}$ and $100 \mathrm{~mm} \mathrm{NaHCO}_{3}, \mathrm{pH}$ 8.0) with rotation at $4^{\circ} \mathrm{C}$ overnight. Beads were washed with $1 \mathrm{ml}$ of coupling buffer, and remaining functional groups were blocked by incubation with $400 \mu \mathrm{l}$ of $0.2 \mathrm{M}$ ethanolamine in coupling buffer at room temperature for $2 \mathrm{~h}$. After preblocking with 3\% BSA and washing with lysis buffer, $20 \mu \mathrm{l}$ aliquots of beads (loaded with 15-30 $\mu \mathrm{g}$ of protein) were incubated with brain lysate as above.

Pull-down assays with recombinant proteins as soluble ligands. GST fusion proteins were expressed and bound to glutathione agarose according to the instructions of the manufacturer. They were not eluted from the affinity matrix after washing but kept under recombinant pull-down buffer (20 mm Tris, pH 8.0, $150 \mathrm{~mm} \mathrm{NaCl}, 1$ mm EDTA, and 1\% Triton $\mathrm{X}-100$ ) with 5\% BSA until use. His-tagged proteins (pQE, pBAD/ThioTOPO, or pET based) were purified on nickel agarose, eluted with 300 $\mathrm{mm} \mathrm{NaCl}, 500 \mathrm{~mm}$ imidazole, $1 \%$ Triton X-100, and $50 \mathrm{~mm} \mathrm{Na}-$ phosphate, $\mathrm{pH} 8.0$, and kept in elution buffer until use on the next day. In a pull-down reaction, typically $10 \mu \mathrm{l}$ packed beads carrying 200-400 pmol of GST fusion protein were incubated with 300-1100 pmol of His-tagged ligand proteins, individually and in combinations added directly in imidazole elution buffer, in $500 \mu$ l total volume [pull-down buffer, adjusted to equal volumes of imidazole elution buffer as introduced by the ligand protein samples $(45-75 \mu \mathrm{l})$ and supplemented with $0.3-0.75 \% \mathrm{BSA}$ ] for $2 \mathrm{~h}$ at room temperature under head-over-end rotation. After removal of the supernatant, beads were washed three times for $90 \mathrm{~s}$ in $1 \mathrm{ml}$ of pull-down buffer without BSA, resuspended in SDS sample buffer, and processed for SDS-PAGE, Coomassie blue staining, and immunoblotting. Pull-down experiments with the thioredoxin Mu1-Mu 5 series (see Fig. 3A) and the thioredoxin Cast3c-1 to Cast3c-9 series (see Fig. $3 B$ ) were performed with crude bacterial lysates of the soluble ligands. C-terminal His-tagged fusion proteins (in pET and $\mathrm{pBAD})$ were detected with anti-His(C-Term) monoclonal antibody (R930-25; Invitrogen) and N-terminal His-tagged fusion proteins (in pQE) with anti-RGS-His monoclonal (34610; Qiagen).

Surface plasmon resonance interaction analysis. The biosensor experiments were performed using a BIAcore 2000 instrument (GE Healthcare). Flow cells on a CM5 chip (GE Healthcare) were activated with a 1:1 mixture of $50 \mathrm{~mm} N$-hydroxysuccinimide and $200 \mathrm{~mm} \mathrm{~N}$-ethyl- $N^{\prime}$ ((dimethylamino)propyl)carbodiimide for $7 \mathrm{~min}$. A polyclonal antiGST antibody (27-4577-01; GE Healthcare) was diluted to $0.1 \mathrm{mg} / \mathrm{ml}$ into $50 \mathrm{~mm}$ sodium acetate, $\mathrm{pH}$, and injected over the surface to produce antibody densities of 10-12 kRU. The surfaces were subsequently blocked with ethanolamine $(1 \mathrm{M}, \mathrm{pH} 8.5)$ and then postconditioned with three 1-min injections of $50 \mathrm{~mm} \mathrm{NaOH}$. All immobilization steps were performed using a flow rate of $5 \mu \mathrm{l} / \mathrm{min}$. Binding experiments were performed at $25^{\circ} \mathrm{C}$ using a running buffer composed of $20 \mathrm{~mm}$ Tris, $\mathrm{pH} 8.0$, $200 \mathrm{~mm} \mathrm{NaCl}, 1 \mathrm{~mm}$ EDTA, and $0.1 \%$ Triton X-100. GST fusion proteins $(0.5 \mathrm{mg} / \mathrm{ml})$ were loaded on the antibody surface for $3 \mathrm{~min}$ at a flow rate of $10 \mu \mathrm{l} / \mathrm{min}$, whereupon His6-Cast3c or Mul:pet was injected for $1 \mathrm{~min}$ at a flow rate of $30 \mathrm{ml} / \mathrm{min}$. For analysis of the ternary complex (see Fig. $4 B, C$ ), an injection of $0.15 \mathrm{mg} / \mathrm{ml} \mathrm{His} 6$-Cast $3 \mathrm{c}$ with a contact time of 2 min was followed by a 1 min injection of Mul:pet. The dissociation was monitored for 5 min before the surface was regenerated with 1 min pulses of $50 \mathrm{~mm}$ glycine, $\mathrm{pH} 2.5$, and $50 \mathrm{~mm} \mathrm{NaOH}$. Analytes were tested at four concentrations using a series of fivefold dilutions. For each experiment, response data were processed using a reference surface to correct for bulk 
refractive index changes and any nonspecific binding. Data were also referenced using responses from blank analyte injections. To determine the binding constants, association and dissociation phase data were globally fitted to a heterogeneous interaction model $\left(\mathrm{A}+\mathrm{B}=\mathrm{AB}, \mathrm{A}^{*}+\mathrm{B}=\right.$ $A^{\star} B$ ) using the Biaevaluation software (GE Healthcare). The presented SDs are based on replicates performed with two different surfaces and protein preparations and sets of three and four concentrations in the global data analysis.

Expression of fluorescent-tagged recombinant proteins and FM dye labeling in hippocampal microcultures, live-cell imaging. Dissociated postnatal rat hippocampal microcultures were prepared as described previously (Brigadski et al., 2005). Primary postnatal [postnatal day 0 (P0) to P2] neocortical astrocytes were isolated and cultured for 2-4 weeks in DMEM containing $10 \%$ fetal calf serum (FCS) until confluence. Astrocytes were passaged and seeded on glass coverslips at a density of 80,000 cells per $3.5 \mathrm{~cm}$ dish in DMEM/10\% FCS, to yield spherical astrocyte islands of 100-300 $\mu \mathrm{m}$ in diameter after 7-14 d in vitro (DIV). Postnatal rat (P0-P2) hippocampal neurons were plated in DMEM/10\% FCS at a density of 1-10 neurons per astrocyte island onto the astrocyte coverslips. After $20 \mathrm{~h}$, the plating medium was exchanged to serum-free medium (Neurobasal with 2\% B27 supplement; Invitrogen).

Cultures were transfected with the respective expression plasmids at 8-9 DIV, using the $\mathrm{Ca}^{2+}$ phosphate precipitation method as described previously (Haubensak et al., 1998). During incubation (3 h), $10 \mu \mathrm{M}$ DNQX and $100 \mu \mathrm{M}$ D,L-APV were added. Cells were used for experiments 1-2 d after transfection (9-11 DIV). The accumulation of EGFP-Acz7-2 and EGFP-Rim5/8 at synapses became visible $12 \mathrm{~h}$ after transfection. However, additional diffuse labeling of the soma, axons, and dendrites was also evident throughout the expression period. Neurons did not tolerate well expression of the fusion constructs beyond $60 \mathrm{~h}$ after transfection. DsRed2-VAMP2 and EGFP-VAMP2 were generated by PCR from a rat VAMP2 cDNA plasmid (Brigadski et al., 2005). To identify presynaptic structures in EGFP-Acz7-2- and EGFP-Rim5/8-expressing hippocampal neurons, cells were cotransfected with DsRed2-VAMP (DNA ratio of EGFP/DsRed, 1:1). Activity-dependent labeling of presynaptic terminals in hippocampal neurons was performed as described previously (Klau et al., 2001; Mohrmann et al., 2003). Briefly, coverslips with hippocampal neurons were incubated for $1 \mathrm{~min}$ at room temperature in HEPES-buffered saline (HBS) containing $50 \mathrm{~mm} \mathrm{~K}^{+}$(replacing an equal amount of $\left.\mathrm{Na}^{+}\right), 2 \mathrm{~mm} \mathrm{Ca}{ }^{2+}, 1 \mathrm{mM} \mathrm{Mg}^{2+}$, and $10 \mu \mathrm{M} \mathrm{FM} 4-64$ [N-(3-triethylammoniumpropyl)-4-(6-(4- diethylamino)phenyl)hexatrienyl)pyridinium dibromide] (Invitrogen). Cells were washed four times in dye-free HBS with low $\mathrm{K}^{+}(4 \mathrm{~mm})$ and without $\mathrm{Ca}^{2+}$ and were transferred into the recording chamber of an upright microscope (BX51WI; Olympus).

Colocalization of EGFP-labeled presynaptic proteins with DsRed2VAMP2 or FM4-64 was investigated with a Nipkow spinning-disk confocal system (Visitech), attached to a conventional fluorescence microscope (BX51WI; Olympus), and equipped with a $60 \times$ waterimmersion objective (numerical aperture 0.90). Glass coverslips with transfected cells were mounted in the recording chamber of the microscope. Full-frame images were taken with a cooled CCD camera (CoolSnap HQ; Roper Scientific). Green and red fluorescence was excited with the 488 and $568 \mathrm{~nm}$ lines of a krypton/argon laser (Laser Physics), respectively. Exposure times were chosen such that saturation was avoided. Image capture and data analysis was performed using MetaMorph software (Molecular Devices ) and Adobe Photoshop software (Adobe Systems), without compromising the evident primary image information. However, in the figures, fluorescence is enhanced close to saturation to facilitate the visibility of synaptic structures. Red and green fluorescent pictures, respectively, were taken from the identical field of view, thresholded, and converted to binary pictures. The percentage of green punctate structures colocalizing with the red fluorescence signal was calculated for each field of view. Average values were determined from at least five independent experiments ( $n>3$ cells per experiment analyzed) for each combination of construct and staining procedure. All calculations were performed with MetaMorph software (Molecular Devices) and Excel (Microsoft).

\section{Results}

\section{Aczonin, Bassoon, CAST, Munc13, and Rim} coimmunoprecipitate from brain lysate

The molecular layout of Aczonin as a giant multidomain protein suggests a role as a scaffolding protein in the molecular organization of the active zone. We therefore probed for binding between Aczonin and other presynaptic proteins by coimmunoprecipitation from brain lysate. The majority of Aczonin cosediments with an insoluble subcellular fraction (Wang et al., 1999; Fenster et al., 2000 ), but $\sim 10 \%$ remain in the $100,000 \times g$ supernatant of a brain lysate, which was used in the following immunoprecipitation and pull-down experiments. Anti-Aczonin antibodies selectively coprecipitated Bassoon, CAST, Munc13, and Rim (Fig. $1 A)$. Conversely, antibodies against Bassoon, Munc13, and Rim coprecipitated the respective other proteins (Fig. $1 B$ ). These results were confirmed with precipitating antibodies against two other sequence regions of Aczonin, a second sequence region of Munc13-1 and a second epitope of Rim1 (Fig. $1 B$, and data not shown). The corresponding preimmune sera and several other antibodies [cadherin, syntaxin, CASK, neurobeachin, palmdelphin, protein kinase A (PKA) subunit RII $\alpha$ ] did not coprecipitate Aczonin, Bassoon, CAST, Munc13, or Rim (Fig. 1A, B, and data not shown).

We probed the immunoprecipitates with antibodies against numerous presynaptic proteins (Fig. $1 A$, and data not shown: synaptophysin, synaptogyrin, synaptobrevin-2, SNAP-25, cadherin, syntaxin, CASK, synapsin-1, Munc18, rabphilin-3, syndapin-1, complexin-2, tomosyn, NSF, $\alpha / \beta$-SNAP, neurobeachin, $\beta$-NAP, and PKA-RII $\alpha$ ) but did not detect additional binding partners. This does not exclude that some of these proteins do bind to Aczonin, Bassoon, Munc13, or Rim but evade detection in this assay. For example, we did not detect the published binding of syntaxin to Munc13 (Betz et al., 1997) or of SNAP-25 to Rim (Coppola et al., 2001) under our conditions. However, we confirmed the coprecipitation of synaptobrevin-2 and tomosyn with anti-syntaxin (Fujita et al., 1998; Hatsuzawa et al., 2003). The observed association between Aczonin, Bassoon, CAST, Munc13, and Rim therefore seems to be very robust. Note, in particular, the strong enrichment of Bassoon and CAST from the lysate by Aczonin immunoprecipitation. These results indicate the existence of a very stable complex encompassing all five proteins that are characteristic for and highly enriched at active zones (in brief, "active zone-specific"): Aczonin, Bassoon, CAST, Munc13, and Rim.

\section{The second coiled-coil domain of Aczonin precipitates Bassoon, a region around the third coiled-coil domain of Aczonin precipitates CAST, Munc13, and Rim, and a region near the Bassoon C-terminus precipitates Munc13 and Rim} Immobilized recombinant partial Aczonin sequences, collectively representing most of the Aczonin molecule (Fig. 1C) (supplemental Table 1, available at www.jneurosci.org as supplemental material), were used in pull-down experiments with brain lysate (Fig. 1D). Aczonin construct Acz5 (amino acids 3059-3336, DIN ... PRN) precipitated native Bassoon, and Aczonin construct Acz7 + 8 (amino acids 3593-4121; SRA ... SSS) precipitated native CAST, Munc13, and Rim. As positive controls, fusion proteins with the zinc-finger domain of Rim1 precipitated Munc13 only (Betz et al., 2001; Wang et al., 2001). Confirming the specificity and avidity of these interactions, no other Aczonin binding partners could be detected in the precipitates with antibodies against numerous additional presynaptic candidate proteins [Aczonin itself, synapsin-1 (Fig. 1D), 

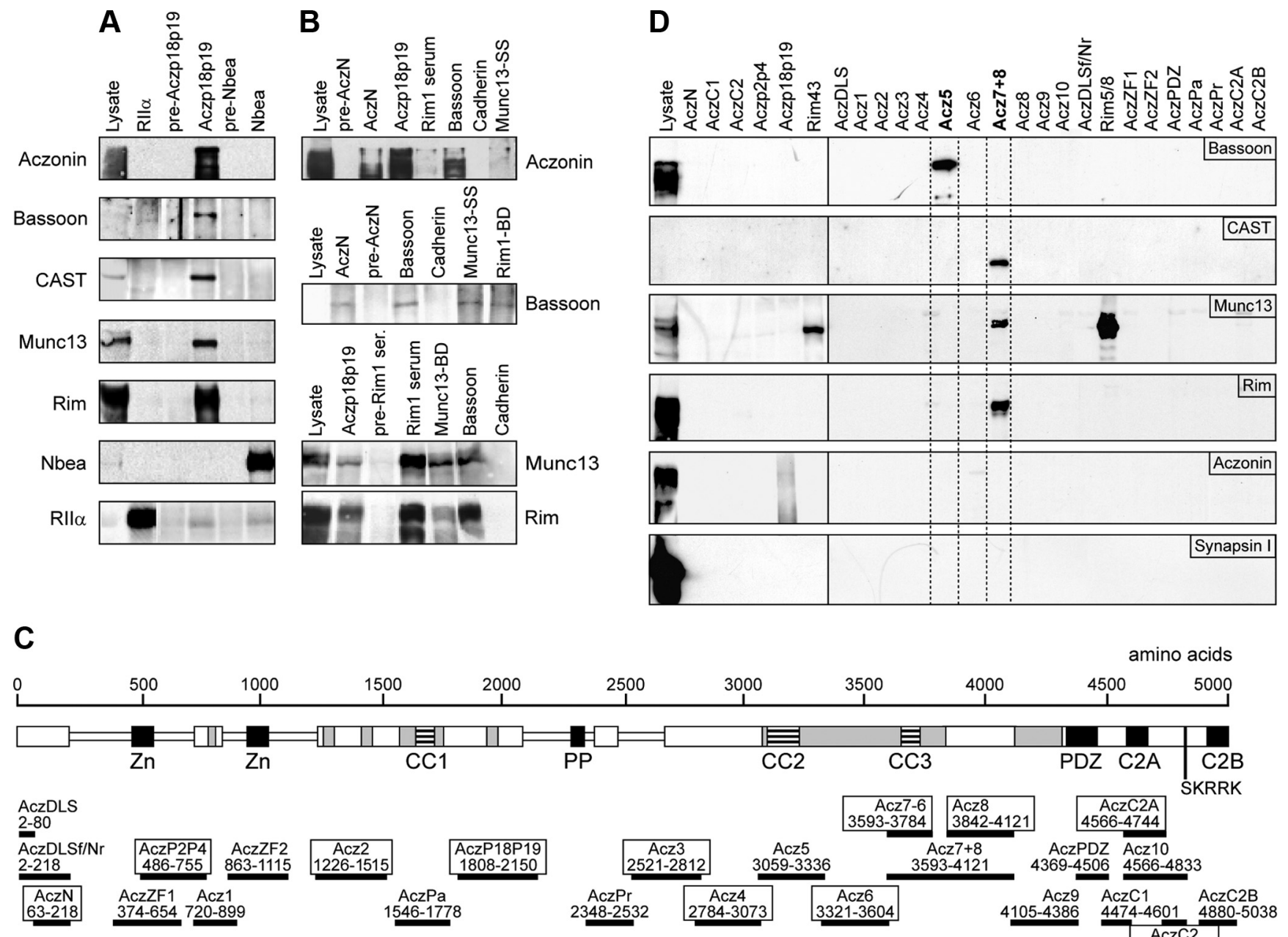

Figure 1. Aczonin binds native Bassoon, CAST, Munc13, and Rim. A, B, Aczonin, Bassoon, CAST, Munc13, and Rim mutually coimmunoprecipitate from brain lysate. Brain lysate was subjected to immunoprecipitation with antibodies against Aczonin, Bassoon, Munc13, Rim1, and controls [preimmune sera and antibodies against cadherin, Nbea and PKA-Rll $\alpha$ ], as indicated at the top, and Western blots of precipitates were probed with antibodies as indicated on the sides. See Materials and Methods for antibody details. In $A$, the weak background signals in the Rll $\alpha$ panel (anti-Acz and anti-Nbea precipitate lanes) are the Ig heavy-chain bands; in the top of $\boldsymbol{B}$, Aczonin signals in the anti-Rim and anti-Munc13 precipitate lanes are weak and do not reproduce well. $\boldsymbol{C}, \mathbf{D}$, The recombinant second coiled-coil region of Aczonin pulls down native Bassoon, and the third coiled-coil region of Aczonin pulls down native CAST, Munc13, and Rim. Aczonin sequence regions were expressed as GST or His-tagged fusion proteins (overview in C, with construct designations and amino acid intervals) and used in pull-down experiments with brain lysate. Pull-down pellets were analyzed by Western blotting $(\boldsymbol{D})$ with antibodies against the proteins indicated on the panels. Pull-down constructs to the left of the vertical line in $\boldsymbol{D}$ were His-tagged fusion proteins immobilized by covalent coupling, and constructs to the right were GST fusion proteins immobilized by glutathione affinity binding. The smear in the Aczp18p19 lane is attributable to residual fusion protein left behind in the gel, because the same Aczonin sequence was used for pull down and for generating the antibody with which the blot was probed; the lane was blank when the blot was reprobed with an antibody against a different Aczonin sequence (data not shown). GST constructs with the Munc13-binding zinc-finger domain of Rim1 (Rim43, Rim5/8) were used as positive controls. Constructs also used for immunization are boxed in $\boldsymbol{C}$. The schematic of the Aczonin molecule represents sequences with low interspecies conservation as narrow bars and sequences with high interspecies conservation as wide bars; sequences partially conserved between Aczonin and Bassoon are shaded. Zing-finger (Zn), coiled-coil (CC1-CC3), polyproline (PP), PDZ, C2A and C2B domains, and the short splice variant ending with a SKRRK amino acid sequence are also indicated.

Munc18, synaptophysin, synaptogyrin, synaptobrevin-2, SNAP25, synaptotagmin, syntaxin, CASK, rabphilin-3, syndapin-1, complexin-2, tomosyn, NSF, $\alpha / \beta$-SNAP, neurobeachin, $\beta$-NAP, cadherin, and PKA-RII $\alpha$ (data not shown)].

The Bassoon-precipitating region of Aczonin was narrowed down to an interval of 125 amino acids (construct Acz5-3, amino acids 3094-3218, VGE ... QYN) coinciding with the second predicted coiled-coil domain of Aczonin (Aczonin-CC2) (Fig. 2A). This sequence is highly conserved between Aczonin and Bassoon. However, although this recombinant Aczonin region pulled down native Bassoon with high avidity and specificity, it did not pull down native Aczonin (Fig. 2A). Neither did the corresponding recombinant Bassoon region, Bsn5, pull down native Bassoon or native Aczonin (Fig. 2A), nor could we detect direct binding between the recombinant Acz5-3 and Bsn5 constructs (data not shown). These negative findings speak against a symmetrical homodimerization or heterodimerization that involves the same coiled-coil domain on both sides. The negative results with the recombinant Bsn5 construct may also be attributable to, e.g., inappropriate boundary design of Bsn5 (although it extends at both ends by 11 or 26 aa beyond the collinear coiled-coil sequence of Acz5-3 highly conserved between Aczonin and Bassoon), to a dependence of the interaction on posttranslational modification of Bassoon, or to a participation of additional proteins from the brain lysate. The molecular details of this Aczonin-Bassoon interaction therefore remain to be worked out.

Figure $2 B$ shows a deletion series in which the Aczonin regions that precipitate native CAST, Munc13, and Rim from brain lysate were narrowed down. CAST bound to a sequence interval of only 59 aa (construct Acz7-3; amino acids 3657-3715, 

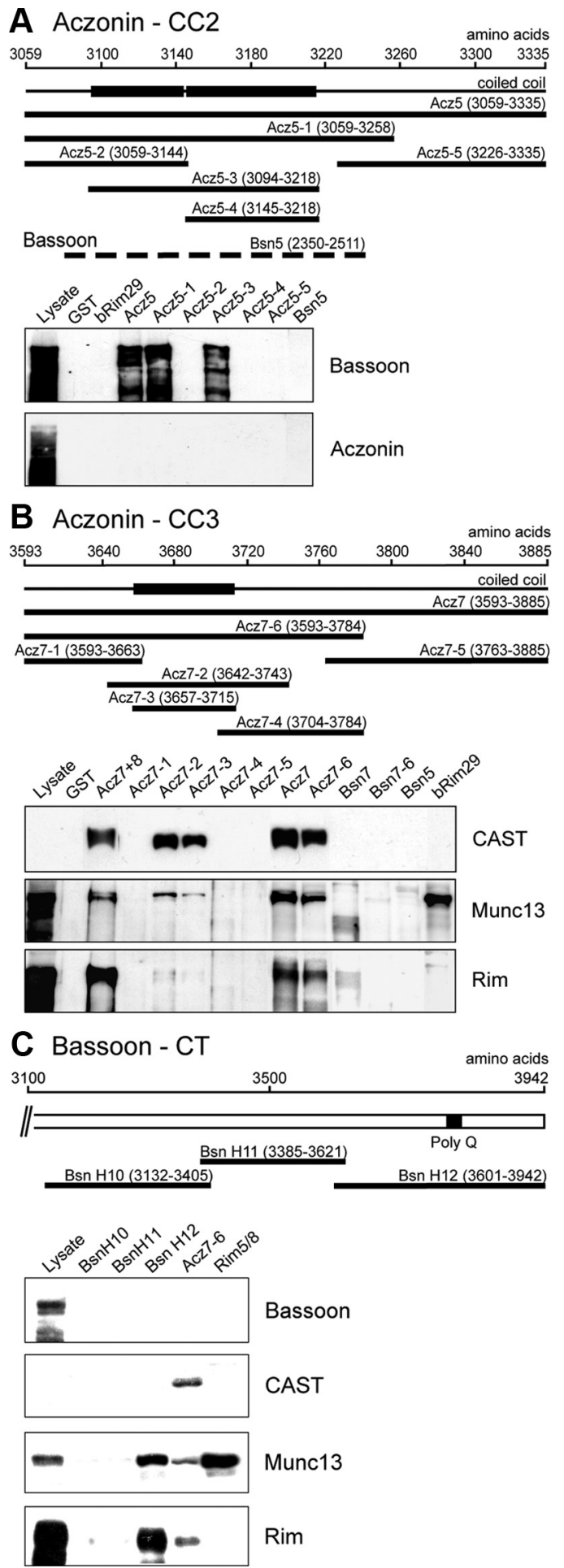

Figure 2. Pull down of native binding partners by the Aczonin- $\mathrm{C} C 2$ and - $\mathrm{C} C 3$ regions and by the Bassoon-CT region. $A, B$, Fine mapping of the Aczonin regions that precipitate native Bassoon, CAST, Munc13, and Rim. Recombinant GST-fused Aczonin sequence intervals (indicated above the panels) around the second and third predicted coiled-coil regions $\mathrm{CC}_{2}(\boldsymbol{A})$ and $\mathrm{CC}(\boldsymbol{B})$, and collinear Bassoon$\mathrm{CC} 2$ and -CC3 sequences, were used for pull down from brain lysate, and Western blots of the pellets were probed with antibodies against Bassoon and Aczonin ( $\boldsymbol{A}$ ) or against CAST1, panMunc13 (antibody Munc13-BD), and $\operatorname{Rim} 1(\boldsymbol{B})$, as indicated on the sides. The broad band in the Bsn7 lane of the Munc13 and Rim panels is background labeling of the GST-Bsn7 fusion protein. The Rim 1 zinc finger constructbRim29 was included as a Munc13-precipitating positive control.C, The C-terminal region of Bassoon precipitates native Munc13 and Rim. Three GST-fused sequence intervals in the C-terminal region of Bassoon were used for pull down from brain lysate, and a Western blot of the pellets was probed with antibodies against Bassoon, CAST1, Munc13, and Rim1. Constructs Acz7-6 and Rim5/8, displaying different ligand specificities, were included as positive controls.
RAK ... ERE) that coincides with the third predicted coiled-coil region of Aczonin (Aczonin-CC3). In contrast, efficient precipitation of Munc13 and Rim required a larger interval around the CAST-precipitating site (construct Acz7-6, amino acids 35933784, SRA... PYT), although weak Rim and Munc13 signals were reproducibly obtained also with the short constructs Acz7-2 and Acz7-3. Of note, the Aczonin constructs precipitated two bands detected by the Munc13 antibody. The lower, more intense band probably is the isoform Munc13-1. It is selectively precipitated also by the Rim1 zinc-finger control construct bRim29, and its binding is primarily but not completely lost in the constructs shorter than Acz7-6. In contrast, precipitation of the upper band, probably a Munc13 splice variant or isoform such as bMunc13-2, is fully retained or even stronger with the shorter constructs Acz7-2 and Acz7-3. The intensity of the Rim pull-down signal essentially parallels that of Munc13, with weak but well reproducible precipitation also by constructs Acz7-2 and Acz7-3. No pull down of native CAST, Munc13, or Rim by the homologous Bassoon sequences Bsn7 and Bsn7-6 (supplemental Table 1, available at www.jneurosci.org as supplemental material) could be detected. We will later discuss the details of these results in the light of binding measurements between recombinant protein domains.

When recombinant GST-Bassoon fusion proteins covering the $\mathrm{C}$-terminal region of Bassoon were used in pull-down experiments with brain lysate (Fig. 2C) (supplemental Table 1, available at www.jneurosci.org as supplemental material), construct BsnH12 (amino acids 3601-3942, SDR ... SFW) was found to precipitate native Munc13 and Rim but not CAST. This sequence region is Bassoon specific (unrelated to the Munc13/Rimprecipitating region Acz7-6) and has no predicted coiled-coil potential.

\section{Demonstration of direct binding and delineation of binding regions}

The third Aczonin coiled-coil region (Aczonin-CC3), the Bassoon $C$-terminal region (Bassoon-CT), and the Rim1 zinc-finger domain (Rim1-ZF) converge on the Munc13-1 N-terminal domain (Munc13-1-NT)

A series of Munc13-1 partial sequences was constructed, as thioredoxin and/or His-tagged fusions collectively covering the entire Munc13-1 protein (Fig. 3A) (supplemental Table 1, available at www.jneurosci.org as supplemental material), for pull-down binding assays with immobilized Aczonin and Bassoon GST fusion constructs. The binding sites of Munc13-1 for Aczonin-CC3 and Bassoon-CT were both found to reside in the N-terminal, 320-aa-long region (construct Mu1) (Fig. 3A) that is also known to bind Rim1-ZF (Betz et al., 2001). Additional Munc13-1 sequences between amino acids 321 and 651 (constructs Mu1 + 2-1 to Munc1 + 2-6) (Fig. 3A) did not strengthen the binding of Munc13-1-NT to immobilized Aczonin-CC3, Bassoon-CT, and Rim1-ZF further [data not shown; tested with GST constructs Acz7-6, the Acz7-3/CAST3c complex, BsnH12, BsnH12-7, and Rim5/8 (see below) as immobilized ligands]. Dissection of region Mul (supplemental Table 1, available at www.jneurosci.org as supplemental material) revealed that full Rim1-ZF-binding activity could be localized to the N-terminal 181 aa of Munc13-1 (constructs Mu1-1 and Mu1-7), but weak Rim1-ZF binding was also seen with constructs Mu1-2 (amino acids 2-102) and Mu1-5 (amino acids 2-143), all in accordance with the observations of $\mathrm{Lu}$ et al. (2006). In contrast, the complete $\mathrm{N}$-terminal 320 aa region of Munc13-1 (construct Mu1) was necessary for full Aczonin- 

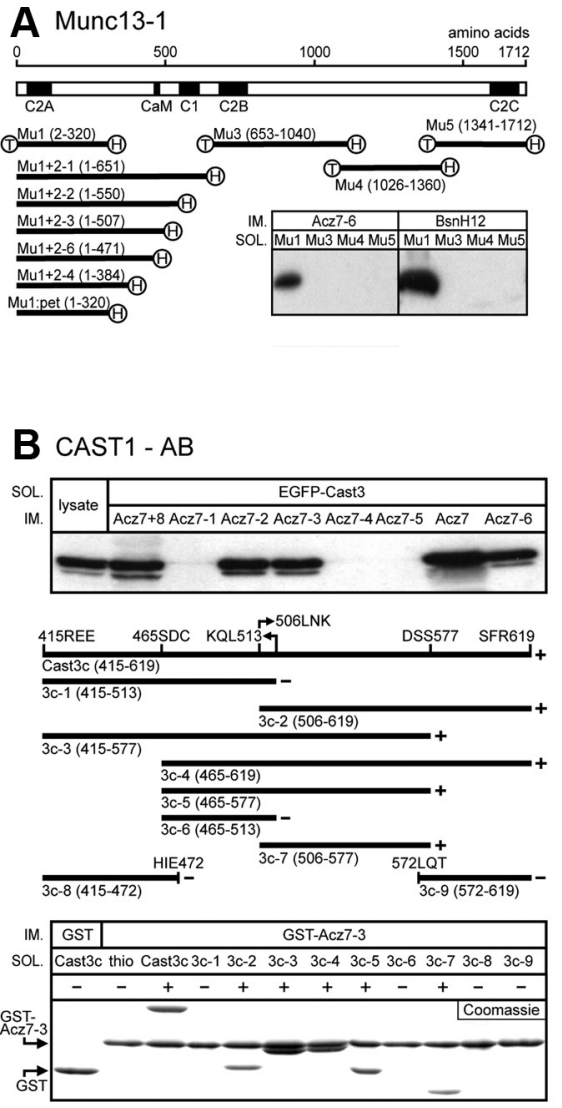

found in the same short Aczonin sequence, Acz7-3, which is also sufficient for binding of native CAST (compare Fig. $3 B$, top, with Fig. $2 B$ ). The Cast 3 sequence was dissected further in the form of bacterially expressed His-tagged and thioredoxin fusion constructs (supplemental Table 1, available at www. jneurosci.org as supplemental material), and the AczoninCC3-binding site of CAST1 ("CAST1$A B$ ”) was finally localized within an interval of only 72 aa (construct Cast3c-7, LNK ... DSS) (Fig. 3B, middle and bottom). Like its binding partner Acz7-3 (59 aa long), this sequence is short and has a high predicted coiled-coil potential. This Aczonin-CAST1 interaction is very robust, producing nearequimolar binding stoichiometries in the Coomassie blue-stained pull-down pellets even after washing (Fig. 3B, bottom).

Composite binding sites in the Aczonin-CC3 region for the Munc13-1 N-terminal domain Pull-down experiments were performed with a series of immobilized GST-Aczonin constructs and $\mathrm{Mul}$ :pet as the soluble ligand (Fig. 3C). Construct Acz7 (amino acids 3593-3885, SRA ... PLM), and apparently also its C-terminally truncated derivatives Acz7-8 and Acz7-7, displayed optimal binding activity within this series. Good but suboptimal binding of the Munc13-1 construct was observed during additional C-terminal truncation to Acz7-6 and Acz7-1 + 3 . Also, Acz7-5 displayed significant Munc13 binding, whereas the sequences N-terminal of amino acid 3593 (Acz7-9, Acz7-10) and between amino acids 3716 and 3784 (Acz7-4, 7-3 + 4) did not detectably contribute to binding. The GST-Acz7-5 construct expressed and bound to glutathione agarose poorly, so that the protein quantity of GSTAcz7-5 on this blot is only $\sim 25 \%$ of the other Aczonin constructs (data not shown), and the immunodetection signal in this lane therefore understates the Mul:pet binding strength of Acz7-5. The GST-Acz7-6 fusion protein migrates as a thick band very closely above the Mul:pet band, displacing it downward and

CC3- and Bassoon-CT-binding activity, although various partial sequences in the Mul region displayed weaker binding of these two ligands (data not shown).

A short coiled-coil binding site of CAST1 for Aczonin-CC3 CAST1 partial sequences collectively representing the complete CAST1/ERC2/ELKS2 protein (constructs Cast1-Cast4) (supplemental Table 1, available at www. jneurosci.org as supplemental material) were expressed as EGFP fusions in COS7 cells and the cell lysates used in pull-down experiments with immobilized GST-Aczonin-CC3 fusion constructs (data not shown). Only construct Cast3 (amino acids 325-677, DNE . . . KEE) bound Aczonin. Full binding activity for recombinant EGFP-Cast3 was probably also quenching part of the Mul:pet immunodetection signal in this lane. In conclusion, an extensive region of Aczonin (Acz7; $293 \mathrm{aa}$ ) around the CC3 sequence is involved in direct binding to Munc13-1-NT, and determinants in both its N-terminal (Acz7-1 + 3; amino acids 3593-3715, SRA ... ERE) and its C-terminal parts (Acz7-5; amino acids 3763-3885, SQF ... PLM) contribute to this binding. The short CC3 sequence alone (construct Acz7-3) displayed no detectable Munc13-1 binding in the present and most other experiments, but weak binding was occasionally observed at high $\mathrm{Mul}$ concentrations, suggesting that it may also contribute determinants (data not shown). 
The Bassoon C-terminal binding site for the Munc13-1 N-terminal domain

A series of immobilized GST-Bassoon fusion constructs (Fig. 3D) (supplemental Table 1, available at www.jneurosci.org as supplemental material) was used in pulldown assays with the recombinant Munc13-1 N-terminal constructs Mu1: pet (amino acids 1-320) and Mul + 2-6 (amino acids 1-471) (shown in Fig. 3D), and also with Mul $+2-4$ (amino acids 1-384) and thioredoxin-Mul (amino acids 2-320) (data not shown). Constructs BsnH12, H12-7, C1, and C3 displayed the strongest binding with no reproducible differences between each other; the binding strengths of $\mathrm{BsnC} 4$ and $\mathrm{C} 5$ were slightly suboptimal; BsnH12-5 bound moderately but clearly suboptimally, and weaker binding was also detected with H12-4 and H12-6 and very weakly with $\mathrm{H} 12-1$. It appears that the C-terminal core binding site of Bassoon for Munc13-1 resides in construct BsnH12-5 (amino acids 3601-3746), with determinants mediating partial binding activity in both halves of this sequence (constructs H12-4, H12-6, and H12-1), but that neighboring sequences either on the N-terminal (construct BsnC1) or the C-terminal side (BsnH12-7) are required for full binding activity (possibly as folding aids). In the experiments of the present study, we mostly used BsnH12-7 as the construct representing the C-terminal Munc13-1-binding site of Bassoon. Very similar binding patterns were obtained with all four Munc13-1 constructs tested, suggesting that Munc13-1 amino acids 321-471 do not significantly contribute to binding to the Bassoon-CT region.

\section{CAST1-AB binds Aczonin-CC3 and Bassoon-CC3 with nanomolar affinity: quantitative characterization by SPR biosensor analysis}

To determine quantitative binding parameters, we studied the identified interactions by a real-time biophysical technique, surface plasmon resonance (SPR) biosensor analysis. Construct His6-Cast3c bound to immobilized GST-Acz7-3, GST-Acz7-6, and GST-Bsn7-6 (the Bassoon partial sequence homologous to Acz7-6; amino acids 2873-3077, AKE ... GSS) (supplemental Table 1 , available at www.jneurosci.org as supplemental material) with qualitatively similar binding kinetics. The nonlinear regression analysis required the assumption of interaction equations containing two exponentials for a reasonable, and three exponentials for an optimal, fit. This is suggestive of a complex, interactive binding mechanism. The results of the two-exponential regression analysis are shown in Figure $4 A$ and Table 1. CAST1-AB binding to the three constructs has faster, low-affinity and slower, high-affinity components, both contributing with similar $R_{\max }$ values to total binding. The ratio between the sum of the two $R_{\max }$ values and the level of preloaded Acz7-3, Acz7-6, or Bsn7-6 after correction for the respective molecular weight approximated a value of 1 , indicating 1:1 binding stoichiometries for CAST1-AB and the three constructs. Acz7-3 and Acz7-6 displayed similar affinities for Cast3c, with $K_{\mathrm{D}}$ values of 7-10 nM for the high-affinity components. Bsn7-6 binding of Cast $3 \mathrm{c}$ was weaker, with a $K_{\mathrm{D}}$ value of $\sim 30 \mathrm{~nm}$ for the high-affinity component. The low-affinity $K_{\mathrm{D}}$ values were approximately one order of magnitude larger than the respective high-affinity values (Table 1).

\section{CAST1-AB also binds directly to Munc13-1-NT and reinforces Aczonin-Munc13-1 binding by forming a ternary complex with both}

Because the Aczonin-CC3 region can bind both CAST1-AB and Munc13-1-NT, with the CAST1-binding sequence nested within a larger area required for Munc13-1 binding, we next explored whether these two binary interactions occur independently or whether they influence each other. Whereas Biacore measurements of Mu1:pet binding to immobilized GST-Acz7-3, GSTAcz7-6, and also GST-Rim5/8 as a positive control produced only weak SPR responses that did not permit a meaningful quantitative evaluation, Figure $4 B$ demonstrates that preloading of immobilized GST-Acz7-6 with His6-Cast3c dramatically stimulated Mul:pet binding, although most of the GST-Acz7-6bound His6-Cast $3 \mathrm{c}$ had been washed out again when Mu1:pet was injected (Fig. 4C).

This ternary Aczonin/CAST1/Munc13-1 interaction was further explored in pull-down experiments (Fig. 5A), in which we additionally included the Rim 1-ZF region (construct Rim5/8) as an established ligand of Munc13-1-NT. In accordance with the results of Figures $2 B$ and $3 C$, GST fusion constructs Acz7-6 and Acz7- $1+3$ pulled down His-tagged $\mathrm{Mu} 1$ and Cast $3 \mathrm{c}$ individually but did not bind Rim5/8 directly. However, in the presence of Cast $3 \mathrm{c}$, the pull down of Mul was substantially enhanced, and, in this case, Rim5/8 was visibly coprecipitated with Mul, suggesting that the binding sites of Rim1-ZF and of the Aczonin/CAST1-AB complex on the surface of Munc13-1-NT do not overlap and that a complex comprising all four partners can be formed. Con- 
Table 1. Quantitative parameters of CAST1-AB binding to the Aczonin- and Bassoon-CC3 sequences, determined by SPR biosensor analysis

\begin{tabular}{|c|c|c|c|c|c|c|c|c|}
\hline & $k_{\mathrm{on} 1}\left(\mathrm{~s}^{-1} \mathrm{~mm}^{-1}\right)$ & $k_{\mathrm{on} 2}\left(\mathrm{~s}^{-1} \mathrm{~mm}^{-1}\right)$ & $k_{\text {off } 1}\left(s^{-1} \times 10^{3}\right)$ & $k_{\text {off } 2}\left(s^{-1} \times 10^{3}\right)$ & $K_{\mathrm{D} 1}(\mathrm{~nm})$ & $K_{\mathrm{D} 2}(\mathrm{~nm})$ & Cast3c: Acz/Bsn & $R_{\max 1} / R_{\max 2}$ \\
\hline Acz7-3 & $128 \pm 76$ & $270 \pm 200$ & $0.78 \pm 0.17$ & $40 \pm 8$ & $6.9 \pm 2.0$ & $240 \pm 200$ & $1.60 \pm 1.03$ & $1.46 \pm 0.44$ \\
\hline Acz7-6 & $119 \pm 56$ & $310 \pm 210$ & $1.03 \pm 0.17$ & $48 \pm 11$ & $10.4 \pm 4.9$ & $210 \pm 120$ & $0.71 \pm 0.31$ & $0.99 \pm 0.19$ \\
\hline Bsn7-6 & $64 \pm 44$ & $250 \pm 110$ & $1.58 \pm 0.19$ & $97 \pm 45$ & $31 \pm 14$ & $380 \pm 120$ & $0.78 \pm 0.31$ & $0.93 \pm 0.23$ \\
\hline
\end{tabular}

On rates $\left(k_{\mathrm{on} 1}, k_{\mathrm{on} 2}\right)$, off rates $\left(k_{\mathrm{off} 1}, k_{\mathrm{off} 2}\right)$, affinities $\left(K_{\mathrm{D} 1}, K_{\mathrm{D} 2}\right)$, and saturation levels $\left(R_{\max 1}, R_{\max 2}\right)$ for a high-affinity $(1)$ and low-affinity (2) interaction were derived from a global two-exponential fit to $S P R$ sensorgrams at different His6-Cast3c concentrations. The binding stoichiometries between Cast3c and Aczonin or Bassoon were calculated as ratios between $\left.\left(R_{\max 1}+R_{\max 2}\right) / \mathrm{MW}_{\text {Cast3c }}\right)$ and the preloaded levels of Acz7-3, Acz7-6, or Bsn7-6 subtracted by the respective molecular weight.

structs Acz7-3 + 4 and Acz7-3 bound only Cast3c but not Mul or Rim5/8 individually (again in accordance with Figs. $2 B, 3 C$ ), but, in the presence of Cast $3 \mathrm{c}$, they also coprecipitated $\mathrm{Mu} 1$ and Rim5/8. The Bassoon-CC3 construct GST-Bsn7-6 displayed qualitatively similar but weaker binding behavior than its homologous GST-Acz7-6 counterpart: binding of Mul alone to GSTBsn7-6 was not detectable in most experiments, Cast3c and $\mathrm{Mu} 1$ together were precipitated but with lower efficiency than by Acz7-6 (concordant with the lower Cast3c affinity of Bsn7-6 measured by SPR) (Table 1 ), and putative Rim5/8 coprecipitation with $\mathrm{Mu} 1$ was below detection. The Cast $3 \mathrm{c}$ chemiluminescence signals in the GST-Bsn7-6 pull down were significantly weaker than in the GST-Acz construct pull downs, as confirmed by Coomassie blue staining (data not shown). The Bassoon-CT construct GST-BsnH12-7 as well as the Rim1-ZF construct GSTRim5/8 bound Mu1 but not Cast3c, and their Mu1 binding was not notably influenced by Cast3c. GST-BsnH12-7 coprecipitated Rim5/8 only in the presence of Mul (suggesting nonoverlapping Bassoon and Rim1 binding interfaces on Munc13-1-NT), whereas Mul binding to GST-Rim5/8 was competitively weakened by His6-Rim5/8.

The results of Figure $5 \mathrm{~A}$ confirm the finding of Figure $3 C$ that determinants flanking the CC3 sequence of Aczonin (represented by constructs Acz7-6 and Acz7-1 + 3) display a moderate direct binding propensity for Munc13-1-NT, whereas the AczoninCC3 sequence itself (Acz7-3 and Acz7-3 + 4) is insufficient to produce a binary binding signal in the pull-down assay. However, in complex with the Cast $3 \mathrm{c}$ sequence, the short Aczonin constructs strongly bind Munc13-1-NT, and also the Munc13-1-NTbinding activity of the long Aczonin constructs is significantly enhanced. Figure $5 B$ uses a CAST1 deletion series and demonstrates that the short His6-Cast3c-7 construct is as efficient as the longer His6-Cast3c-5 and His6-Cast3c constructs not only in binding to GST-Acz7-3 (confirming the result of Fig. $3 B$ ) but is also fully sufficient to mediate the coprecipitation of Mul:pet. Note again the very efficient, near-stoichiometric formation of the Aczonin/Cast complex demonstrated by Coomassie blue staining. The Mul:pet protein bands migrate very closely below the GST-Acz7-6 bands and can be seen on the Coomassie bluestained gel but are not resolved in reproduction; immunoblot detection of Mul:pet is therefore additionally shown at the top. A weaker Mu1:pet band was also precipitated by GST-Acz7-6 (but not by GST-Acz7-3) in the absence of Cast constructs, visible on the Coomassie blue-stained gel but below the immunoblot detection threshold.

The coprecipitation of Munc13-1-NT by the Aczonin-CC3/ CAST1-AB complex could be attributable to (1) an allosteric activation, by CAST1 binding, of a Mul-binding propensity of this Aczonin sequence, (2) the constitution of a composite Mu1-binding interface to which both Acz7-3 and Cast3c-7 synergistically contribute determinants, (3) the existence of independent, Acz7-3-binding and Mul-binding sites within the Cast3c-7 sequence, or a combination of these mechanisms. To investigate this, we incubated immobilized GST-Mul with the
His-tagged Cast $3 c$, Cast $3 c-5$, and Cast $3 c-7$ proteins alone and indeed observed robust (Coomassie blue staining), direct binding of all three CAST1 sequences with similar efficiencies to the Munc13-1 N-terminal region (Fig. 5C). Hence, the same 72-aashort Cast $3 \mathrm{c}-7$ sequence binds independently and avidly to both Aczonin-CC3 and Munc13-1-NT, reinforcing the AczoninMunc13-1 interaction that is additionally mediated, independent of CAST1, by Aczonin sequences flanking Acz7-3.

\section{A complex of domains from all five active-zone-specific proteins centers on the N-terminal region of Munc13-1}

At this point, it had become clear that domains from the five active-zone-specific proteins Aczonin, Bassoon, CAST1, Munc13-1, and Rim 1 converge on a node of interactions that centers on the $\mathrm{N}$-terminal domain of Munc13-1. We therefore performed pulldown experiments with GST-Mul as the immobilized binding partner and the His- or thioredoxin-tagged Acz7-3, BsnH12-7, Cast $3 c$, and Rim5/8 proteins as ligands in solution. In this constellation of fusion constructs, the Bassoon, CAST1, and Rim1 constructs can all bind directly to the immobilized Munc13-1 construct and were efficiently precipitated in stoichiometries readily visible through Coomassie blue staining (Fig. 5D). They bound individually and in all combinations with very similar efficiencies, indicating neither synergy nor competition. However, because all binding stoichiometries were significantly below 1 (at least after washing; they may have been close to 1 at equilibrium during the incubation), we cannot exclude that competition between some partners for overlapping binding sites on, or for space around, Munc13-1-NT may have gone undetected in this assay. Acz7-3 was coprecipitated only in the presence of Cast3c, confirming the results of Figure $5, A$ and $B$, in reverse fusion construct orientation. In the constellation based on a GST$\mathrm{Mu} 1$ fusion construct, however, the binding stoichiometry of thioredoxin-Acz7-3 (and likewise of untagged Acz7-3 cleaved from GST-Acz7-3 with thrombin; data not shown) via Cast3c was low and had to be detected by immunoblotting, unlike the more efficient binding of Mu1:pet to GST-Acz7-3/Cast complexes (Fig. 5B). We attribute this to sterical or folding interference by the N-terminal GST fusion moiety of Mul. The experiment shown used the dimerization-mutant GST-Mu1(K32E) construct, but identical results were obtained with wild-type GST$\mathrm{Mul}$ as the immobilized partner. At the right of Figure $5 D$, we show the results of two binary pull downs between immobilized GST-Mu1(K32E) and either wild-type or K32E-mutant Mu1: pet. Homodimerization was abolished only if both constructs carried the K32E mutation reported by Lu et al. (2006), whereas significant, although suboptimal, binding was retained when only one of the two constructs was K32E mutant. An immunoblot is shown because the Mul:pet protein bands are obscured by proteolytic fragments of GST-Mu1(K32E). The schematic model of Figure $5 E$ summarizes the findings of the preceding experiments and integrates them with previously determined interactions between Aczonin, Bassoon, CAST1, Munc13-1, and Rim. The model also shows known molecular features in the vicinity of 

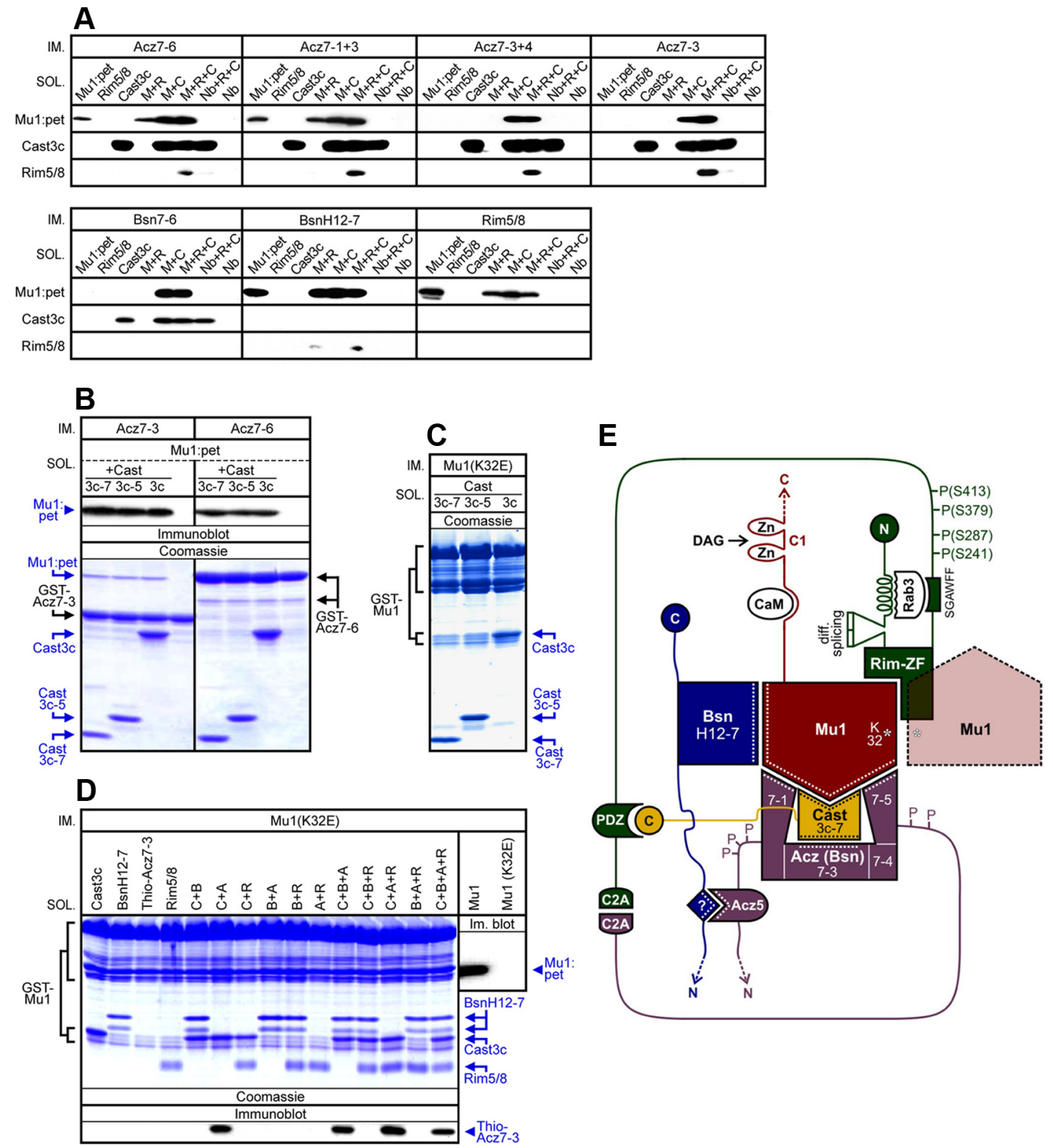

Figure 5. Munc13-1-NT forms hetero-oligomeric complexes with Aczonin/Bassoon-CC3, Bassoon-CT, CAST1-AB, and Rim1-ZF and binds directly to CAST1-AB. A, CAST1-AB reinforces binding between the Aczonin or Bassoon CC3 regions and Munc13-1-NT, leading to a quaternary complex including Rim1-ZF. Immobilized GST fusion proteins (IM.) representing the Aczonin- and Bassoon-CC3 regions (GST-Acz7-X, Bsn7-6), the Bassoon-CT region (BsnH12-7), and the Rim1-ZF region (Rim5/8) (Figs. 2, 3) (supplemental Table 1, available at www.jneurosci.org as supplemental material) were incubated with His-tagged proteins in solution (SOL.) representing the Munc13-1-NT (Mu1:pet), CAST1-AB (His6-Cast3c), and Rim1-ZF (His6-Rim5/8) regions, individually or in combinations. As a negative control, a His-tagged protein from the PKA-Rll binding region of neurobeachin (Nb; construct Nbea-C2) (Wang et al., 2000) was also included; no unspecific pull down of this control protein was detected (data not shown). Pull-down pellets were analyzed by Western blotting with antibodies against N-terminal (detecting Cast3c, Rim5/8, and Nbea-C2) and C-terminal His tags (Mu1:pet) as shown and also by Coomassie blue staining (data not shown). All pull-down experiments of the type shown included a full set of negative controls (data not shown) in which the same solutions of soluble ligands, in all combinations used in the binding assays, were incubated in parallel with beads carrying insert-less GST to exclude unspecific binding or precipitation. B, The 72-aa-short CAST1 sequence Cast3C-7 is sufficient to reinforce Munc13-1-NT binding to Aczonin-CC3. Pull downs were performed with immobilized GST-Acz7-3 and GST-Acz7-6 and with Mu1:pet alone or in combination with His6 -Cast3C, His6 —Cast3C-5, or His6 -Cast3C-7 in solution. All CAST1 constructs bound the Aczonin-CC3 constructs with similar, 1:1 stoichiometries, and stimulated Mu1:pet pull down. C, The 72-aa-short CAST1 sequence Cast3C-7 binds directly to Munc13-1-NT. A Coomassie blue-stained gel of a pull down with immobilized GST-Mu1 (dimerization-deficient mutant K32E) demonstrates that all CAST1 constructs bind with similarly high efficiencies directly to Munc13-1-NT. The GST-Mu1 fusion protein is isolated with a series of proteolytic fragments in addition to the full-length protein at the top, as indicated by brackets at the left margins of $\mathbf{C}$ and $\boldsymbol{D}$; the full-length band accounts for $>50 \%$ of the total protein, and its intense staining relative to the proteolytic fragments is underrepresented in reproduction. D, Aczonin-CC3, Bassoon-CT, CAST1-AB, and Rim1-ZF converge on Munc13-1-NT. Pull-down assays were performed with immobilized GST-Mu1(K32E), and His6-BsnH12-7, His6-Cast3c, His6-Rim5/8, and Thio-Acz7-3-His6 as ligands in solution, individually and in all possible combinations. The Bassoon, CAST1, and Rim1 constructs bound with very similar efficiencies in all combinations, readily detectable by Coomassie blue staining. His 6 -BsnH12-7 appears as a double band; the upper band is the full-length protein, and the lower band is a proteolytic fragment truncated by $13-17$ aa at the $\mathrm{N}$ terminus (determined by mass spectrometry). The His 6 -Nbea- $\mathrm{C} 2$ negative control protein (see $\boldsymbol{A}$ ) did not bind (data not shown). The binding efficiency of thioredoxin-Acz7-3 to the GST-fused Mu1 construct was low and only detectable by immunoblotting with an antibody against its C-terminal His tag (shown at the bottom). Direct binding, to GST-Mu1, of Acz7-6 or other longer Aczonin constructs could not be investigated because the His-tagged Aczonin constructs were poorly expressed and insoluble, and the insert of GST-Acz7-6 was degraded by thrombin. $\boldsymbol{E}$, Schematic summary of (1) the binding constellations between the Aczonin/Bassoon-CC3, Bassoon-CT, CAST1-AB, Munc13-1-NT, and Rim1-ZF regions, including homodimerization of Munc13-1-NT, which is known to compete with Rim-ZF binding; (2) known sites of potential modulatory signal input in the vicinity of these regions; and (3) interactions between other regions of these five active zone proteins that add to the interconnectivity, identified in the present study (Aczonin-CC2 domain to unknown features of Bassoon, possibly indirectly through a third partner) or as published previously [CAST ( terminus to Rim PDZ domain (Ohtsuka et al., 2002; Wang et al., 2002), C2A domains (Figure legend continues.) 

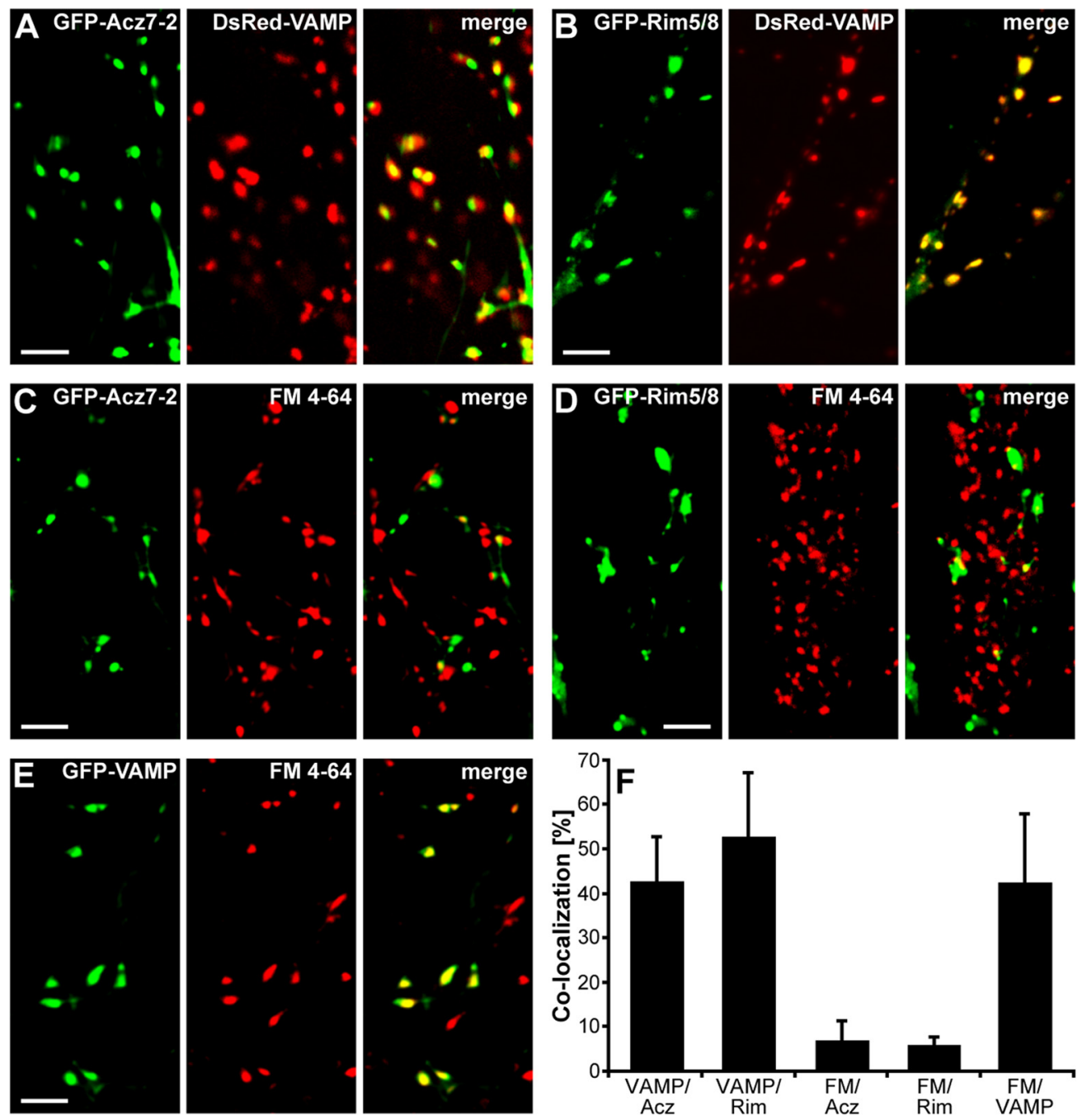

Figure 6. Presynaptic targeting and synaptic silencing by recombinant Aczonin-CC 3 and Rim-ZF sequences. Hippocampal microcultures were transfected at $8-9$ DIV with fluorescent-tagged proteins as indicated and were stained with FM4-64 and analyzed at 9-11 DIV. A-E, Green, red, and merged fluorescent pictures for identical fields of view, respectively, for each panel. $\boldsymbol{F}$, Quantification of the percentage of green fluorescence colocalizing with red fluorescence in the same field of view $(n>5$ independent experiments for each construct, $n>3$ cells per experiment analyzed). Whereas, in approximately half of the green fluorescent spots, the Acz and Rim constructs colocalized with the presynaptic marker DsRed-VAMP2, a negligible portion ( $<6 \%$ for each construct) of these synapses was able to cycle vesicles. In contrast, $>40 \%$ of GFP-VAMP2-labeled synapses revealed vesicle cycling (significantly different from EGFP-Acz7-2 and EGFP-Rim5/8, with $p<0.001)$. Scale bars, $5 \mu \mathrm{m}$.

the interacting domains that may provide regulatory input (see legend and Discussion).

Aczonin-CC3 and Rim1-ZF are targeted to synaptic terminals and interfere with synaptic vesicle turnover

As a first step in analyzing the roles of the identified protein interactions in the ontogeny and functioning of synapses, plasmids encoding EGFP fusion constructs of various domains were expressed in primary rat hippocampal neurons in microisland cultures. One or $2 \mathrm{~d}$ after transfection, the subcellular localization of the expressed fusion proteins was analyzed compared with

(Figure legend continued.) of Aczonin and Rim2 (Fujimoto et al., 2002)]. Interaction-mediating protein domains are shown as space-filling symbols, interfaces of binding newly identified in the present study are marked by dotted lines, and polypeptide intervals between domains are indicated as thin continuous lines. The Aczonin-C2A domain can also homodimerize (Gerber et al., 2001). Known interactions with additional scaffolding proteins such as spectrin, GIT1, syntenin-1, or liprin- $\alpha$ add to the complexity of this network but are not included in this scheme (see Introduction and end of Discussion). cotransfected DsRed-VAMP2. This construct has been reported previously to be efficiently targeted to neurotransmitter vesicles at synaptic structures (Brigadski et al., 2005) and was used as a marker of presynaptic vesicle clusters. To detect active vesicle turnover at synapses, we used activity-dependent labeling of presynaptic structures with the styryl dye FM4-64 (Klau et al., 2001; Mohrmann et al., 2003). The constructs EGFP-Acz7-2 and EGFP-Rim5/8 both decorated patterns of spots along neurites which exhibited a high degree of colocalization with DsRedVAMP2 (Fig. $6 A, B$ ). However, spots positive for EGFP-Acz7-2 and EGFP-Rim5/8 were rarely labeled by FM4-64 (Fig. $6 C, D$ ), in contrast to the positive control in which a high degree of colocalization of EGFP-VAMP2-positive spots (i.e., vesicle clusters, "structural synapses") and FM4-64 (i.e., active vesicle turnover, "functional synapses") was observed (Fig. $6 E$ ). Figure $6 F$ gives the morphometric analysis of colocalization. Accordingly, $\sim 50 \%$ of green fluorescent spots in EGFP-Acz7-2- and EGFP-Rim5/8expressing neurons can be identified as structural synapses. However, once targeted to synaptic terminals, these constructs inhibit vesicle turnover and thus presynaptic function. 
These findings suggest that both Acz7-2 (this sequence binds CAST-AB but not, by itself, Munc13-1-NT) (Figs. $2 B, 3 C$ ) and Rim5/8 (this sequence binds Munc13-1-NT but not Rab3) are efficiently targeted to synaptic terminals and impair vesicle turnover, presumably by inhibiting exocytosis through dominantnegative replacement of holo-Aczonin and holo-Rim from their binding sites on CAST1 and Munc13-1, respectively.

\section{Discussion}

\section{All five active-zone-specific multidomain proteins are multiply interconnected}

In the present study, we have identified several new interactions between domains of proteins of the presynaptic active zone. We show that the Munc13-1 N-terminal domain, in addition to its established homodimerization and to its heterodimerization with the Rim zinc-finger domain (Lu et al., 2006), binds a region near the Bassoon $\mathrm{C}$ terminus, a short sequence stretch in the middle of CAST1, and determinants around the third coiled-coil domain of Aczonin. Moreover, high-affinity binding of the Aczonin-CC3 sequence to CAST1 considerably strengthens, indirectly, the adherence of Aczonin to Munc13-1-NT. An additional connection between Aczonin and Bassoon, whose molecular details remain to be worked out, is mediated by the second coiled-coil domain of Aczonin. Our pull-down results with recombinant proteins (Fig. 5D) suggest that Aczonin-CC3, Rim-ZF, CAST1-AB, and Bassoon-CT can bind simultaneously to Munc13-1-NT (model of Fig. 5E). However, whereas the occurrence of a quaternary complex of Aczonin-CC3, CAST1-AB, Munc13-1-NT, and Rim1-ZF, and of a ternary complex of Bassoon-CT, Munc13-1-NT, and Rim1-ZF, seem established (Fig. 5A), it requires additional clarification whether or not Bassoon-CT competes with CAST1-AB or Aczonin-CC3 for binding to Munc13-1-NT. Munc13-1-NT homodimerization and its heterodimerization with Rim-ZF have been shown previously to compete ( $\mathrm{Lu}$ et al., 2006), and it remains to be investigated whether Aczonin-CC3, CAST1-AB, or Bassoon-CT interfere with the homodimerization of Munc13-1-NT. These findings demonstrate that the five large multidomain proteins of the active zone, Aczonin, Bassoon, CAST, Munc13, and Rim, are closely and multiply interconnected, physically and presumably also functionally. In particular, the organization of this complex around the N-terminal domain of Munc13-1 emphasizes a key role of this domain in the orchestration of molecular events at the active zone. Although Rab3-Rim and Rim-Munc13-1 binding have been known for many years, no downstream effector mechanisms have been identified for them. Our results now place these binary interactions into a larger and much more elaborate molecular context (Fig. 5E), which may be essential for additional progress in mechanistic understanding.

The Bassoon-CC3 domain was reported previously to bind CAST1 (Takao-Rikitsu et al., 2004), and the shortest CAST1binding Bassoon sequence described there (BsnCasBD) is almost precisely collinear and has high sequence similarity with our minimal CAST1-binding Aczonin construct, Acz7-3. However, in SPR and pull-down measurements between recombinant ligands, we find that CAST1 binds more strongly to Aczonin-CC3 than to Bassoon-CC3 (Figs. 4A, 5A; Table 1). Moreover, in our brain lysate pull-down experiments, we detected no precipitation of native CAST1, Munc13, or Rim by the GST-Bsn7/Bsn7-6 constructs, whereas the collinear GST-Aczonin constructs produced strong CAST1, Munc13, and Rim signals in the same experiments (Fig. $2 B$ ). Even long overexposure of the Western blot of Figure $2 B$ showed no trace of CAST1 binding to Bsn7 or Bsn7-6, along- side massive CAST1 signals in the Acz7/7-6 lanes (detection limit: $\sim 1 \%$ of CAST 1 binding to the Aczonin constructs). We note that Takao-Rikitsu et al. (2004) describe precipitation by BsnCasBD only for recombinant, heterologously overexpressed CAST1, not for native CAST1 from a brain lysate. The approximately threefold lower affinity of Bassoon-CC3, relative to Aczonin-CC3, for CAST1-AB measured by SPR (Table 1) thus translates into a significantly poorer performance in the pull down of recombinant CAST1 (Fig. 5A) and a complete failure to pull down native CAST1 from brain lysate, at low ligand concentration and in competition with the native Aczonin in the lysate, suggesting that this affinity difference may be functionally significant.

The CAST1-binding domain of Aczonin (construct Acz7-3) and the Aczonin- and Munc13-1-binding domain of CAST1 (construct Cast3c-7) are small (maximally 60-70 aa each) and engage robustly in a 1:1 complex in vitro. In contrast, the other protein binding modules characterized here are large (Munc131-NT, 320 aa; Bsn-CT, 220-310 aa; the Aczonin region around CC3 capable of Munc13 binding without CAST1, 230-293 aa), and their dissection produces fragments with partial binding activities. Their complexes are substoichiometric in the pull-down assay and give weak or undetectable signals in SPR. This suggests that the respective binding interfaces are extensive and complex, composed of several determinants and dependent on optimal folding.

In light of the binding data with the recombinant proteins, we can now reinspect the details of the pull down of holoproteins from brain lysate (Fig. 2 B). Holo-CAST1 was precipitated by the short and long Acz-CC3 constructs with similar efficiencies. In contrast, Munc13-1 precipitation was strong only with the long constructs, whereas the short Aczonin-CC3 constructs produced only weak Munc13-1 signals. This may reflect a weak residual Munc13-1-binding activity of Acz7-3 alone (which we observed in some recombinant pull-down experiments), or a small fraction of the immobilized Acz7-3 construct (present in vast excess over the individual native proteins in the lysate) has acquired high Munc13-1 affinity through forming a complex with native CAST1 from the brain lysate. Of note, the second Munc13immunopositive band of slower electrophoretic migration precipitated by the Aczonin constructs, but not by the recombinant Rim1-ZF construct bRim29, is pulled down with equal or even higher efficiency by the short than by the long Acz-CC3 constructs. This suggests that this ligand, presumably a Munc13 splice variant or isoform such as bMunc13-2, is bound with full strength also by the short Acz-CC3 constructs. The identity of this second Munc13-like ligand and the details of its mode of binding to Acz7-3 remain to be elucidated.

We interpret the pull down of Rim from brain lysate by the Aczonin-CC 3 and Bassoon-CT constructs (Figs. 1D, $2 B, C$ ) to be indirect, primarily via the Munc13-1 N-terminal region, which can be demonstrated in pull-down experiments with the recombinant constructs (Fig. 5A). Binding between the CAST1 C terminus and the Rim PDZ domain (Ohtsuka et al., 2002; Wang et al., 2002) may additionally contribute to an indirect pull down of Rim from brain lysate, in particular by the short Aczonin-CC3 constructs Acz7-2 and Acz7-3, which bind Munc13-1 poorly. It remains possible that $\operatorname{Rim} 1$ sequences downstream of the Munc13- and Rab3-binding regions (amino acids 1-228) also bind directly to the Acz7 region or other partners of the complex. Fusion proteins representing these Rim 1 sequences were poorly soluble and did not allow us to conclusively answer this question.

The close encounter of domains of Aczonin, Bassoon, CAST1, Munc13-1, and Rim1 in a "node" of interactions suggests a high 
potential for crosstalk. Their juxtaposition may poise these or other domains of their respective holoproteins for fast and precise multilateral or sequential interactions. At least the binary interaction of Aczonin with Munc13-1-NT is profoundly reinforced by the presence of CAST1, which binds avidly to both. Moreover, the domains participating in this interaction node are neighbored by multiple molecular features that may mediate regulatory signal input (Fig. 5E). The Aczonin and Bassoon CC3 sequences are flanked on both sides by in vivo phosphorylation sites (Collins et al., 2005). Munc13-1 carries a Ca ${ }^{2+} /$ calmodulin (CaM) binding site at mouse amino acids 456-473 (Xu et al., 1998; Junge et al., 2004) and a diacylglycerol/phorbol ester binding C1 zinc-finger domain at amino acids 564-614 (Betz et al., 1998). Amino acids 19-50 and the SGAWFF motif around tryptophan 201 of mouse Rim1 interact with Rab3-GTP (Sun et al., 2001; Wang et al., 2001; Dulubova et al., 2005), amino acids 56-82 and 83-105 of Rim 1 can be deleted individually or together by differential splicing (Wang et al., 2001), and phosphorylation has been detected at Rim1 serines 241, 287, and 413 and a Rim2 residue corresponding to Rim1 serine 379 (Lonart et al., 2003; Sun et al., 2003). The Rim1-Rab3 interaction is thought to be involved in the synaptic vesicle life cycle, and the S413 phosphorylation of Rim1 and the interactions of Munc13 with $\mathrm{CaM}$ and diacyl glycerol in synaptic plasticity. For none of these molecular modifications or interactions of Aczonin, Bassoon, Munc13, and Rim, however, have downstream effector mechanisms been identified. It is tempting to suspect that some of the interactions described here might be influenced by them. The C-terminal half of Munc13-1, in particular, is responsible for the synaptic vesicle priming activity of the protein, which in turn is thought to be regulated by signal input through the $\mathrm{N}$-terminal half (Basu et al., 2005; Stevens et al., 2005). Our findings add three new molecular interactions of the Munc13-1 N-terminal half (with CAST1, Aczonin-CC3, and Bassoon-CT) to those already known (with itself, Rim-ZF, $\mathrm{Ca}^{2+} /$ calmodulin, and diacylglycerol), significantly expanding the scope of its regulatory potential. The identification of the multidomain complex described here provides a basis for additional, in-depth analysis of its structural organization, of its molecular dynamics and regulatory modulation, and for selectively addressing the roles of individual binary interactions in neurotransmission.

\section{Involvement of interacting domains in synaptogenesis and synaptic activity}

Figure 6 demonstrates that the Acz7-2 and Rim5/8 fusion proteins with EGFP (1) are targeted to and concentrated at synaptic terminals and (2) suppress synaptic vesicle turnover, suggesting functions of the Aczonin-CC3/CAST1-AB and the Rim1-ZF/ Munc13-1-NT interactions in both the assembly and activity of synapses. The suppression of vesicle turnover by our Rim5/8 construct is consistent with the $\sim 50 \%$ reduction of neurotransmitter release that was achieved by instillation into the calyx of Held synaptic terminal, of a ZF domain from the Rim $2 \alpha$ isoform closely collinear with our Rim1 construct (Dulubova et al., 2005). The effect of Acz7-2 on vesicle turnover is in line with the $~ 50 \%$ suppression of synaptic transmission that Takao-Rikitsu et al. (2004) observed within $100 \mathrm{~min}$ after microinjection into superior cervical ganglion cells, of a CAST1-binding GST-Bassoon fusion protein almost exactly collinear with our Acz7-3 sequence (which in turn is nested within Acz7-2). Takao-Rikitsu et al. (2004) also inhibited synaptic transmission by microinjecting a recombinant CAST1 sequence that binds Aczonin-CC3 or Bassoon-CC3 but also, as we show here for the first time,
Munc13-1-NT. Because of the density of binding interfaces in this multidomain complex, future functional experiments will have to address the various binary interactions individually.

The presynaptic targeting and vesicle turnover suppressing activities of our constructs may occur by "acute" displacement of the endogenous holoprotein in the terminal, particularly during activity; in the course of the in situ turnover of the active zone proteins [which can have time constants in the range of minutes (Munc13-1) (Kalla et al., 2006) to hours (Bassoon) (Tsuriel et al., 2009)], during the de novo assembly of active zone material between the Golgi complex and nascent synapses, or a combination of these mechanisms. Assembly of the active zone machinery, in association with membranes, begins as early as at the Golgi complex or the trans-Golgi network (Wang et al., 1999; Dresbach et al., 2006), followed by dispatch as a coat on dense-core secretory vesicles or multi-vesicle aggregates toward the neuronal periphery in which it can be rapidly deposited at nascent synapses (Ahmari et al., 2000; Zhai et al., 2001; Shapira et al., 2003; TaoCheng, 2007). The hierarchy of protein associations in the course of this assembly is an interesting question, but, given the highly interwoven structure of this macromolecular aggregate, it may not be a simple linear algorithm with molecular "leaders" and "followers." With the respective other four active-zone-specific proteins alone, Aczonin, Bassoon, CAST, and Rim are connected through at least two separate domains each [Aczonin-CC2 and -CC3, Bassoon-CC3 and -CT, CAST1-AB (binding both Aczonin-CC3 and Munc13-NT) and -CT, and Rim-ZF and -PDZ], the Munc13-1-NT binds all four partners, and homodimerization is known for Munc13-1-NT and CAST1 (Fig. 5E). The interaction between Munc13-NT and Rim-ZF, for example, is important but not exclusively responsible for the active zone recruitment of Munc13 (Andrews-Zwilling et al., 2006). Interactions with additional multivalent scaffolding proteins such as those of Rim and CAST1 with liprin- $\alpha$ (Schoch et al., 2002; Ko et al., 2003; Dai et al., 2006), of CAST1 with syntenin-1 (Ko et al., 2006), of Munc13 with $\beta$-spectrin (Sakaguchi et al., 1998), and of Aczonin with GIT1 (Kim et al., 2003) add additional layers of complexity to the molecular architecture of the active zone and to the formation of a polymeric, highly interconnected protein lattice. Hence, the "targeting code" of active zone proteins is probably combinatorial, and, through their early association along the secretory pathway, they can find the way to synaptic terminals as fellow travelers, although each of them may possess only part of the targeting information.

\section{References}

Ahmari SE, Buchanan J, Smith SJ (2000) Assembly of presynaptic active zones from cytoplasmic transport packets. Nat Neurosci 3:445-451.

Andrews-Zwilling YS, Kawabe H, Reim K, Varoqueaux F, Brose N (2006) Binding to Rab3A-interacting molecule RIM regulates the presynaptic recruitment of Munc13-1 and ubMunc13-2. J Biol Chem 281:19720-19731.

Basu J, Shen N, Dulubova I, Lu J, Guan R, Guryev O, Grishin NV, Rosenmund C, Rizo J (2005) A minimal domain responsible for Munc13 activity. Nat Struct Mol Biol 12:1017-1018.

Betz A, Okamoto M, Benseler F, Brose N (1997) Direct interaction of the rat unc-13 homologue Munc13-1 with the $\mathrm{N}$ terminus of syntaxin. J Biol Chem 272:2520-2526.

Betz A, Ashery U, Rickmann M, Augustin I, Neher E, Südhof TC, Rettig J, Brose N (1998) Munc13-1 is a presynaptic phorbol ester receptor that enhances neurotransmitter release. Neuron 21:123-136.

Betz A, Thakur P, Junge HJ, Ashery U, Rhee JS, Scheuss V, Rosenmund C, Rettig J, Brose N (2001) Functional interaction of the active zone proteins Munc13-1 and RIM1 in synaptic vesicle priming. Neuron 30:183-196.

Brigadski T, Hartmann M, Lessmann V (2005) Differential vesicular target- 
ing and time course of synaptic secretion of the mammalian neurotrophins. J Neurosci 25:7601-7614.

Collins MO, Yu L, Coba MP, Husi H, Campuzano I, Blackstock WP, Choudhary JS, Grant SGN (2005) Proteomic analysis of in vivo phosphorylated synaptic proteins. J Biol Chem 280:5972-5982.

Coppola T, Magnin-Luthi S, Perret-Menoud V, Gattesco S, Schiavo G, Regazzi R (2001) Direct interaction of the Rab3 effector RIM with $\mathrm{Ca}^{2+}$ channels, SNAP-25, and synaptotagmin. J Biol Chem 276:32756-32762.

Dai Y, Taru H, Deken SL, Grill B, Ackley B, Nonet ML, Jin Y (2006) SYD-2 liprin- $\alpha$ organizes presynaptic active zone formation through ELKS. Nat Neurosci 9:1479-1487.

Dresbach T, Torres V, Wittenmayer N, Altrock WD, Zamorano P, Zuschratter W, Nawrotzki R, Ziv NE, Garner CC, Gundelfinger ED (2006) Assembly of active zone precursor vesicles: obligatory trafficking of presynaptic cytomatrix proteins Bassoon and Piccolo via a trans-Golgi compartment. J Biol Chem 281:6038-6047.

Dulubova I, Lou X, Lu J, Huryeva I, Alam A, Schneggenburger R, Südhof TC, Rizo J (2005) A Munc13/RIM/Rab3 tripartite complex: from priming to plasticity? EMBO J 24:2839-2850.

Fenster SD, Chung WJ, Zhai R, Cases-Langhoff C, Voss B, Garner AM, Kaempf U, Kindler S, Gundelfinger ED, Garner CC (2000) Piccolo, a presynaptic zinc finger protein structurally related to Bassoon. Neuron 25:203-214.

Fujimoto K, Shibasaki T, Yokoi N, Kashima Y, Matsumoto M, Sasaki T, Tajima N, Iwanaga T, Seino S (2002) Piccolo, a $\mathrm{Ca}^{2+}$ sensor in pancreatic $\beta$-cells - Involvement of cAMP-GEFII. Rim2.Piccolo complex in cAMP-dependent exocytosis. J Biol Chem 277:50497-50502.

Fujita Y, Shirataki H, Sakisaka T, Asakura T, Ohya T, Kotani H, Yokoyama S, Nishioka H, Matsuura Y, Mizoguchi A, Scheller RH, Takai Y (1998) Tomosyn: a syntaxin-1-binding protein that forms a novel complex in the neurotransmitter release process. Neuron 20:905-915.

Gerber SH, Garcia J, Rizo J, Südhof TC (2001) An unusual C2 domain in the active-zone protein piccolo: implications for $\mathrm{Ca}^{2+}$ regulation of neurotransmitter release. EMBO J 20:1605-1619.

Gundelfinger ED, Kessels MM, Qualmann B (2003) Temporal and spatial coordination of exocytosis and endocytosis. Nat Rev Mol Cell Biol 4:127-139.

Hatsuzawa K, Lang T, Fasshauer D, Bruns D, Jahn R (2003) The R-SNARE motif of tomosyn forms SNARE core complexes with syntaxin 1 and SNAP-25 and down-regulates exocytosis. J Biol Chem 278:31159-31166.

Haubensak W, Narz F, Heumann R, Lessmann V (1998) BDNF-GFP containing secretory granules are localized in the vicinity of synaptic junctions of cultured cortical neurons. J Cell Sci 111:1483-1493.

Junge HJ, Rhee JS, Jahn O, Varoqueaux F, Spiess J, Waxham MN, Rosenmund C, Brose N (2004) Calmodulin and Munc13 form a $\mathrm{Ca}^{2+}$ sensor/effector complex that controls short-term synaptic plasticity. Cell 118:389-401.

Kalla S, Stern M, Basu J, Varoqueaux F, Reim K, Rosenmund C, Ziv NE, Brose N (2006) Molecular dynamics of a presynaptic active zone protein studied in Munc13-1-enhanced yellow fluorescent protein knock-in mutant mice. J Neurosci 26:13054-13066.

Kim S, Ko J, Shin H, Lee JR, Lim C, Han JH, Altrock WD, Garner CC, Gundelfinger ED, Premont RT, Kaang BK, Kim E (2003) The GIT family of proteins forms multimers and associates with the presynaptic cytomatrix protein Piccolo. J Biol Chem 278:6291-6300.

Klau M, Hartmann M, Erdmann KS, Heumann R, Lessmann V (2001) Reduced number of functional glutamatergic synapses in hippocampal neurons overexpressing full-length TrkB receptors. J Neurosci Res 66:327-336.

Ko J, Na M, Kim S, Lee JR, Kim E (2003) Interaction of the ERC family of RIM-binding proteins with the liprin- $\alpha$ family of multidomain proteins. J Biol Chem 278:42377-42385.

Ko J, Yoon C, Piccoli G, Chung HS, Kim K, Lee JR, Lee HW, Kim H, Sala C, Kim E (2006) Organization of the presynaptic active zone by ERC2/ CAST1-dependent clustering of the tandem PDZ protein syntenin-1. J Neurosci 26:963-970.

Lonart G, Schoch S, Kaeser PS, Larkin CJ, Südhof TC, Linden DJ (2003) Phosphorylation of RIMla by PKA triggers presynaptic long-term potentiation at cerebellar parallel fiber synapses. Cell 115:49-60.
Lu J, Machius M, Dulubova I, Dai H, Südhof TC, Tomchick DR, Rizo J (2006) Structural basis for a Munc13-1 homodimer to Munc13-1/RIM heterodimer switch. PLoS Biol 4:e192.

Mohrmann R, Lessmann V, Gottmann K (2003) Developmental maturation of synaptic vesicle cycling as a distinctive feature of central glutamatergic synapses. Neuroscience 117:7-18.

Ohtsuka T, Takao-Rikitsu E, Inoue E, Inoue M, Takeuchi M, Matsubara K, Deguchi-Tawarada M, Satoh K, Morimoto K, Nakanishi H, Takai Y (2002) CAST: a novel protein at the cytomatrix at the active zone of synapses that forms a ternary complex with RIM1 and Munc13-1. J Cell Biol 158:577-590.

Sakaguchi G, Orita S, Naito A, Maeda M, Igarashi H, Sasaki T, Takai Y (1998) A novel brain-specific isoform of $\beta$-spectrin: isolation and its interaction with Munc13. Biochem Biophys Res Commun 248:846-851.

Schoch S, Gundelfinger ED (2006) Molecular organization of the presynaptic active zone. Cell Tissue Res 326:379-391.

Schoch S, Castillo PE, Jo T, Mukherjee K, Geppert M, Wang Y, Schmitz F, Malenka RC, Südhof TC (2002) RIM1 $\alpha$ forms a protein scaffold for regulating neurotransmitter release at the active zone. Nature 415:321-326.

Shapira M, Zhai RG, Dresbach T, Bresler T, Torres VI, Gundelfinger ED, Ziv NE, Garner CC (2003) Unitary assembly of presynaptic active zones from Piccolo-Bassoon transport vesicles. Neuron 38:237-252.

Stevens DR, Wu ZX, Matti U, Junge HJ, Schirra C, Becherer U, Wojcik SM, Brose N, Rettig J (2005) Identification of the minimal protein domain required for priming activity of Munc13-1. Curr Biol 15:2243-2248.

Sun L, Bittner MA, Holz RW (2001) Rab3a binding and secretionenhancing domains in Rim1 are separate and unique. Studies in adrenal chromaffin cells. J Biol Chem 276:12911-12917.

Sun L, Bittner MA, Holz RW (2003) Rim, a component of the presynaptic active zone and modulator of exocytosis, binds 14-3-3 through its N-terminus. J Biol Chem 278:38301-38309.

Takao-Rikitsu E, Mochida S, Inoue E, Deguchi-Tawarada M, Inoue M, Ohtsuka T, Takai Y (2004) Physical and functional interaction of the active zone proteins, CAST, RIM1, and Bassoon, in neurotransmitter release. J Cell Biol 164:301-311.

Tao-Cheng JH (2007) Ultrastructural localization of active zone and synaptic vesicle proteins in a preassembled multi-vesicle transport aggregate Neuroscience 150:575-584.

Tsuriel S, Fisher A, Wittenmayer N, Dresbach T, Garner CC, Ziv NE (2009) Exchange and redistribution dynamics of the cytoskeleton of the active zone molecule Bassoon. J Neurosci 29:351-358.

Wang X, Kibschull M, Laue MM, Lichte B, Petrasch-Parwez E, Kilimann MW (1999) Aczonin, a 550-kD putative scaffolding protein of presynaptic active zones, shares homology regions with Rim and Bassoon and binds profilin. J Cell Biol 147:151-162.

Wang X, Herberg FW, Laue MM, Wüllner C, Hu B, Petrasch-Parwez E, Kilimann MW (2000) Neurobeachin: a protein kinase A-anchoring, beige/Chediak-Higashi protein homolog implicated in neuronal membrane traffic. J Neurosci 20:8551-8565.

Wang X, Hu B, Zimmermann B, Kilimann MW (2001) Rim1 and Rabphilin-3 bind Rab3-GTP by composite determinants partially related through N-terminal $\alpha$-helix motifs. J Biol Chem 276:32480-32488.

Wang Y, Liu X, Biederer T, Südhof TC (2002) A family of RIM-binding proteins regulated by alternative splicing: implications for the genesis of synaptic active zones. Proc Natl Acad Sci U S A 99:14464-14469.

Wojcik SM, Brose N (2007) Regulation of membrane fusion in synaptic excitation-secretion coupling: speed and accuracy matter. Neuron 55: $11-24$.

Xu XZ, Wes PD, Chen H, Li HS, Yu M, Morgan S, Liu Y, Montell C (1998) Retinal targets for calmodulin include proteins implicated in synaptic transmission. J Biol Chem 273:31297-31307.

Zhai RG, Vardinon-Friedman H, Cases-LanghoffC, Becker B, Gundelfinger ED, Ziv NE, Garner CC (2001) Assembling the presynaptic active zone: a characterization of an active zone precursor vesicle. Neuron 29:131-143.

Ziv NE, Garner CC (2004) Cellular and molecular mechanisms of presynaptic assembly. Nat Rev Neurosci 5:385-399. 